- This study proposes a simplified method of assessing lingual nerve injuries subsequent to third molar surgery.

- Two point discrimination sensitivity and neuropathic area were the only conventional tests to reliably identify nerve injury.

- The subjective function score may identify patients at risk of permanent injury in the early post-operative period allowing expedition of their referral for specialist care.

\title{
Simplifying the assessment of the recovery from surgical injury to the lingual nerve
}

\author{
T. Renton, ${ }^{1}$ A. Thexton, ${ }^{2}$ S-J. Crean ${ }^{3}$ and M. Hankins ${ }^{4}$
}

\begin{abstract}
Objective To determine the sensitivity of conventional sensory assessment in monitoring lingual nerve recovery subsequent to third molar surgery and to evaluate if the assessment methods can be predictive of injury outcome.

Method A prospective case series of 94 patients presenting with lingual nerve injuries evaluated using objective mechanosensory and subjective methods during the recovery period of up to 12 months.

Results The conventional tests were often unable to diagnose the presence of injury due to variability and they were not predictive of outcome. As a result of this study, we are able to identify patients more likely to have permanent rather than temporary lingual nerve injury at four to eight weeks post injury, using patient reported subjective function. The subjective function test also minimises the requirements for specialist training or equipment providing an ideal method for general dental practice.

Conclusions The development of these simple subjective tests may enable us to identify which patients are at risk of permanent lingual nerve injuries in the early post injury phase, thus allowing expeditious therapy when indicated.
\end{abstract}

\section{INTRODUCTION}

Lingual nerve injuries associated with mandibular third molar surgery are mostly temporary. Eighty-eight per cent resolve in the first four weeks post-operatively, ${ }^{1}$ and it is not presently possible to distinguish neuropraxic from severe axonotmesis or neurotmesis injuries in the early post injury phase.

Scant data are available on the nature and patterns of lingual nerve recovery early after iatrogenic injuries, ${ }^{2-4}$ or later at six months post-operatively. ${ }^{1,5,6}$ As a consequence there is limited information on how and when these injuries resolve and very few reports attempt to identify the predictive value of various tests for

\footnotetext{
${ }^{1 *}$ Professor, Department of Oral Surgery, Dental Institute, King's College London, Denmark Hill Campus, Bessemere Rd, London SE5 9RW; ${ }^{2}$ Reader, Department of Physiology, Dental Institute, King's College, London SE1 9RT; ${ }^{3}$ Consultant Oral and Maxillofacial Surgeon, West Glamorgan NHS Trust; ${ }^{4}$ Senior Statistician, Institute of Psychiatry and Psychology, King's College, London SE1 9RT

*Correspondence to: Professor Tara Renton

Email: tara.renton@kcl.ac.uk
}

\section{Refereed paper}

Accepted 9 May 2005

doi: $10.1038 /$ sj.bdj.4813584

๑ British Dental Journal 2006; 200: 569-573 lingual nerve injury resolution. ${ }^{6}$ Mason $^{1}$ stated that the most important information that the patient wishes to know is will the neuropathy recover, and if so, when? Currently, reassurance is based on the probability of recovery, as most cases do recover spontaneously. However, 20 years later we are no closer to solving the prediction conundrum and there is still no consensus on which tests should be used for the assessment of these injuries, or whether the patient's opinion should be considered foremost. There is a need to establish a simple formula of nerve assessment that may identify patients at risk of permanent injury, which may result in earlier referral to specialist practitioners.

Subjective tests may not comprehensibly evaluate sensory discriminatory capacity. However, they provide a quantification of the location and spatial extent of sensory alteration, and thereby provide a basis for selection of sites for further clinical assessment. Only two studies report detailed longitudinal assessment of the outcome of lingual nerve injuries. ${ }^{1,6}$ Most of the studies evaluating conventional mechanical tests have been performed on patients with inferior alveolar nerve injuries subsequent to orthognathic surgery. A recent study reports that brush stroke direction, warm/cold and sharp blunt tests are unreliable in evaluating inferior alveolar nerve injury. ${ }^{7}$ Others agree there are significant limitations of these mechanosensory tests. ${ }^{8}$ Due to their variability, these tests in the oro-facial regions have proved to be unhelpful ${ }^{9,10}$ and do not correlate with assessed physical damage at surgical exploration. ${ }^{11}$ Light touch and two point discrimination appear to have the greatest 'objectivity' yet often do not agree with the patient's subjective assessment. For example, patients reporting abnormal sensory function may only display abnormal clinical test results in 50\% of cases ${ }^{11}$ and significant discrepancies exist between the altered sensation reported by patients and the neurosensory abnormalities detected by clinical tests. ${ }^{4,12,13}$ The measurement of taste is unreliable following injury of the lingual nerve ${ }^{14,15}$ as taste perception is reported to be normal in patients with known nerve injury. ${ }^{15}$ As a result, assessment of taste function was omitted in the current study.

There is a need for further investigation of lingual nerve injuries in order to clarify the relationship between the patient reported sensory alteration and clinical neurosensory testing. ${ }^{13}$ Gregg ${ }^{16}$ has stated that current methods of stimulus detection after trigeminal nerve injury are an inadequate measure of sen- 
after trigeminal nerve injury are an inadequate measure of sensory deficiency or potential for recovery. It may be possible that a formula could be developed in order to define the patient's progress towards recovery. This includes the following three domains: functional impairment (mastication, hygiene, speech, work, sleep, social interaction), patient perceived abnormality and modality stimulus detection (A beta fibre - fine and crude touch, noxious, electrical stimulus thresholds; A delta - cooling thresholds and C fibres warm and noxious heat thresholds). This concept has already been successfully applied to hand surgery. ${ }^{17}$

This prospective study was undertaken to identify the most appropriate and clinically applicable methods of assessing the extent of injury to the lingual nerve. A primary objective was to establish if these tests were capable of identifying the presence of a lingual nerve injury and a secondary objective was to assess if any of these tests could distinguish permanent from temporary in the early post injury phase.

\section{SUBJECTS AND METHODS}

Ninety-four consecutive patients were seen in Guy's Dental School Oral and Maxillofacial Surgery Department with lingual nerve injuries subsequent to mandibular third molar surgery. Patients with medical conditions that could compromise neurosensory function were excluded from this study. Out of over 200 patients seen in the department, 94 patients with lingual nerve injury were evaluated at two, four, eight, 12, 16, 24 weeks and 12 months until the injury had resolved or reparative surgery was carried out.

\section{Setting}

Patients provided consent in accordance with the local ethical committee, and were then questioned and examined by a single examiner (to avoid inter-operator variability) at each visit. All subjects were tested in a draught free and silent room, at an ambient room temperature, reclining in a dental chair.

\section{Sensory testing}

Several investigations were made at each sitting; these included: identifying the area affected, symptoms (paraesthesia, anaesthesia, dysaesthesia), two-point discrimination, light touch perception threshold (Semmes Weinstein monofilaments RDG Medical, UK.), sharp blunt discrimination, fungiform papillae count (FPC), palpation of the lingual nerve in the retromolar region and a subjective functional report. Mechanosensory testing was performed in the middle of the neuropathic area which was usually situated $1 \mathrm{~cm}$ lateral to the midline and $1.5 \mathrm{~cm}-2 \mathrm{~cm}$ back from the tip of the tongue (Fig. 1) and compared with a matched reference site selected on the contralateral side. Each test was carried out in random order, on alternating sides.

\section{Symptoms}

The patients were asked to describe any altered sensations, including paraesthesia, anaesthesia or dysaesthesia (allodynia, hperalgesia) that they considered were associated with the injury. Further information concerning the character of the altered sensation was sought, including intensity, onset (evoked/ spontaneous, precipitating factors), relief, frequency (intermittent or constant) and whether it was troublesome.

\section{Area affected by hypoaesthesia}

A number 12 monofilament in light contact with the lingual mucosa was moved into and away from the neuropathic region and the area of neuropathy was recorded on a proforma. Using a transparent grid this area was then converted to a percentage of the tongue hemi-surface. Trauma or scarring due to tongue biting was recorded separately.

\section{Two-point discrimination threshold (2PDT)}

2PDT thresholds were obtained with blunt probes calibrated in millimetres on keys around a key ring. 2PDT was applied, without movement on the mucosa. Testing started with orientation of the callipers $2 \mathrm{~mm}$ apart followed by a gradual increase in interprobe distance by $1 \mathrm{~mm}$ until the patient reported two points by showing one or two fingers. The threshold was estimated at the distance that was reported correctly in three out of five events.

\section{Light touch pressure sensibility}

Perception threshold (Semmes Weinstein monofilaments) was estimated using the method of limits in ascending series. Each of the 20 filaments varies in diameter (range 0.06-1.14 mm) for which is assigned a calibration value corresponding to a $\log _{10}$ of the strength required to bend the filament into a demi-circle. For threshold detection, successive fibres were applied until perception of the stimulus was established. The threshold was recorded for five applications ${ }^{18}$ and the thresholds were calculated by taking the mean of the stimuli values perceived.

\section{Sharp or blunt discrimination (S/B)}

A sharp right angle dental probe was applied to the lingual area, with indentation but with no breach of the mucosa, the patient was then asked to compare this sensation to that produced by the sharp or blunt region of the probe. The test was considered positive if the patient recognised three out of five of each stimulus correctly.

\section{Fungiform papillae count (FPC)}

FPC were obtained by eye while placing a key on the tested area with a cut out area of $1 \mathrm{~cm}^{2}$, at a site $1 \mathrm{~cm}$ from the midline 1.5 $\mathrm{cm}$ back from the tongue tip on the right and left sides (Fig. 1).

\section{Palpation of the lingual nerve in the retromolar region}

The retromolar area was palpated to ascertain if there was pain or unpleasantness associated with finger pressure in the lingual nerve region. This has been reported to be associated with neuroma formation and indicative of permanent injury. ${ }^{18}$

\section{Subjective function}

The contralateral side to the injury was stimulated with a No. 12 filament providing $50 \mathrm{~g} / \mathrm{mm}^{2}$ pressure (Semmes Weinstein monofilaments) and the patient was asked to imagine that this was equivalent to 10/10 compared to nothing touching the area, which would be equivalent to $0 / 10$. The injured side of the tongue was then stimulated with the same pressure and repeated bilaterally until the patient could report a value (VAS score 0-

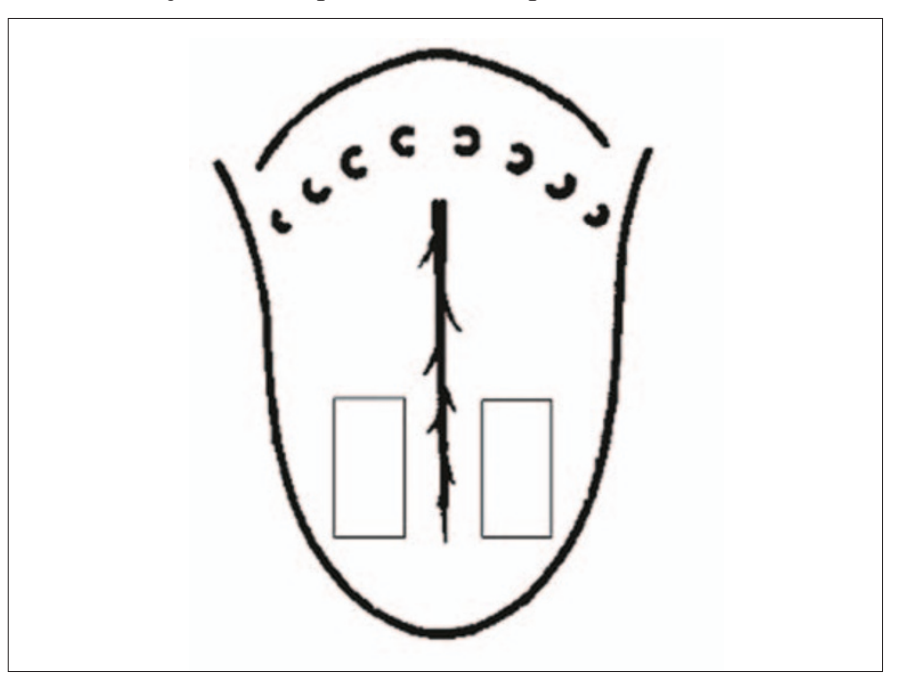

Fig. 1 Sites selected for clinical examination of mechanosensory and subjective lingual nerve function. 


\begin{tabular}{|c|c|c|c|c|c|c|c|}
\hline Variable & $\begin{array}{l}2 \\
\text { weeks }\end{array}$ & $\begin{array}{l}4 \\
\text { weeks }\end{array}$ & $\begin{array}{l}8 \\
\text { weeks }\end{array}$ & $\begin{array}{l}12 \\
\text { weeks }\end{array}$ & $\begin{array}{l}16 \\
\text { weeks }\end{array}$ & $\begin{array}{l}24 \\
\text { weeks }\end{array}$ & $\begin{array}{l}52 \\
\text { weeks }\end{array}$ \\
\hline TI group $n=$ & 42 & 44 & 21 & 8 & 4 & 4 & 0 \\
\hline PI group $n=$ & 6 & 7 & 8 & 9 & 7 & 10 & 14 \\
\hline Age & 0.146 & & & & & & \\
\hline Gender & 0.085 & & & & & & \\
\hline Ethnicity & 0.134 & & & & & & \\
\hline Anaesthetic & 0.718 & & & & & & \\
\hline $\begin{array}{l}\text { surgeon } \\
\text { grade }\end{array}$ & 0.73 & & & & & & \\
\hline Method & 0.683 & & & & & & \\
\hline Pain & 0.004 & & & & & & \\
\hline $\begin{array}{l}\text { Subject } \\
\text { function }\end{array}$ & 0.006 & $<0.001$ & 0.006 & $<0.001$ & $<0.001$ & $<0.001$ & 1 \\
\hline 2PDT injured & 0.182 & 0.001 & 0.009 & 0.248 & 0.353 & 0.092 & 1 \\
\hline \multicolumn{8}{|c|}{ 2PDT contra 0.795} \\
\hline LT injured & 0.585 & 0.03 & 0.02 & 0.152 & 0.449 & 0.376 & 1 \\
\hline LT contra & 0.6 & & & & & & \\
\hline$S / B$ & 0.938 & 0.15 & 0.407 & 0.485 & 0.818 & 0.271 & \\
\hline $\begin{array}{l}\text { Altered } \\
\text { sensation }\end{array}$ & 0.158 & 0.095 & 0.345 & 0.096 & 0.6 & 0.67 & \\
\hline Scarring & 0.342 & nil & 0.85 & nil & 0.428 & 0.512 & 1 \\
\hline FPC injured & 0.121 & 0.688 & 0.083 & 0.138 & 0.112 & 0.029 & 1 \\
\hline FPC contra & 0.284 & & & & & & \\
\hline Area & 0.136 & 0.001 & 0.009 & $<0.001$ & 0.165 & 0.003 & \\
\hline
\end{tabular}

10) that would best quantify their sensory experience in comparison with the matched contralateral uninjured side.

\section{Data analysis}

Using SPSS (Version 10) bilateral equality of the readings was determined using $t$-tests with the Bonferroni modification and the reproducibility of the recordings was assessed with Pearson's correlation coefficient (r). Those variables that were significant at the univariate level were further tested using multiple regression analysis ANOVA. The Odds Ratios were calculated for those factors shown to be predictive of injury outcome. Data are given as mean (+/-SD) with $\mathrm{P}<0.01$ being considered as statistically significant.

\section{RESULTS}

The ability of the sensory tests to evaluate the presence of injury (Table 1), the ability of the tests to distinguish between temporary and permanent injury (Table 2) and the correlation of mechanosensory tests with subjective function (Table 3) are displayed.

When patient demographic factors were analysed and compared between the permanently injured group (PI group) and temporarily injured groups (TI group), no statistical differences were found for age, ethnicity, gender, surgical method or anaesthesia (Table 1).

\section{Resolution}

Eighty patients had temporary injury and 14 had permanent lingual nerve injury. Temporary injuries resolved at different rates, 34 patients reported their nerve injury recovered within one day to two weeks and 46 resolved by six months. The maximum rate of resolution occurred in the first two weeks and by four weeks $71.5 \%$ of temporary injuries had resolved.

\section{Neuropathic area}

Throughout the review period the area affected by neuropathy was greater in the permanent injury group compared with the temporary injury group (Table 2). The rate of resolution of the area was not predictive for resolution. The lateral border was the
Table 2 Tests able to distinguish between permanent and temporary lingual nerve injured sides ( $P$ values by paired sample $t$-tests).

\begin{tabular}{|c|c|c|c|c|c|c|c|}
\hline & $\begin{array}{l}2 \\
\text { weeks }\end{array}$ & $\begin{array}{l}4 \\
\text { weeks }\end{array}$ & $\begin{array}{l}8 \\
\text { weeks }\end{array}$ & $\begin{array}{l}12 \\
\text { weeks }\end{array}$ & $\begin{array}{l}16 \\
\text { weeks }\end{array}$ & $\begin{array}{l}24 \\
\text { weeks }\end{array}$ & $\begin{array}{l}52 \\
\text { weeks }\end{array}$ \\
\hline \multicolumn{8}{|c|}{ Sub Func } \\
\hline$n=$ & 50 & 51 & 29 & 17 & 11 & 14 & 14 \\
\hline$P=$ & $<0.001$ & $<0.001$ & 0.0017 & $<0.001$ & $<0.001$ & $<0.001$ & $<0.001$ \\
\hline \multicolumn{8}{|c|}{ Fungiform Papillae Count } \\
\hline$n=$ & 48 & 51 & 29 & 17 & 11 & 14 & 14 \\
\hline$P=$ & $<0.001$ & $<0.001$ & 0.002 & $<0.001$ & 0.016 & 0.01 & 0.021 \\
\hline \multicolumn{8}{|c|}{ Light Touch } \\
\hline$n=$ & 50 & 51 & 29 & 17 & 11 & 14 & 14 \\
\hline$P=$ & $<0.001$ & 0.018 & 0.45 & 0.01 & 0.17 & 0.068 & 0.072 \\
\hline \multicolumn{8}{|c|}{ Two Point Discrimination } \\
\hline$n=$ & 50 & 51 & 29 & 17 & 11 & 14 & 14 \\
\hline$P=$ & $<0.001$ & $<0.001$ & 0.004 & 0.001 & 0.003 & $<0.001$ & 0.022 \\
\hline
\end{tabular}

final area to resolve in 36 cases and persisted in all permanent cases.

\section{Symptoms}

The incidence of altered sensation did not differ in the permanent injury group compared with the temporary injury group at any time post-injury (Table 2). However dysaesthesia was found only in the permanent injury group and troublesome altered sensation was more prevalent in this group due to increased reported rates of pain.

\section{Subjective report of function}

Subjective function reliably identified lingual nerve injury in both groups. Resolution of subjective function in the temporary injury group and persistence within the permanent injury group occurred over the review period. In the temporary injury group the score returned to a score of 10 at six months, while the mean subjective function for the permanent injury group remained between 1.6 and 1.9. One exception was a patient who suffered from severe allodynia at week eight post-operatively and thus was inclined to give higher scores than 10 when stimulated. This one patient in the permanent injury group illustrates a possible limitation of this subjective function method, and for those patients suffering from hyperpathia or allodynia, the subjective score may be elevated rather than reduced despite severe injury.

Subjective function significantly differed between the temporary injury and permanent injury groups at two, four, eight, 12, 16 and 24 weeks post-operatively (Table 2). Correlation of patients' reported subjective function with light touch threshold, two-point discrimination, altered sensation, fungiform papillae and neuropathic area was evident at most post-operative periods. The strongest correlation of the subjective function report occurred with the neuropathic area and two point discrimination (Table 3). This test also discriminated between the injured and contralateral side at two, four, 12, 16, 24 and 52 weeks (Table 1) illustrating improved sensitivity compared with the two point discrimination, light touch, sharp/blunt discrimination and fungiform papillae count tests.

\section{Two-point discrimination (2PDT)}

The injured side had significantly greater 2PDT distances than the contralateral sides in the same subject at two to 24 weeks (Table 1). A significant difference between the two-point threshold sensitivity between the two injured groups occurred two to 24 weeks, rendering it as a good method to identify injury (Table 1). A significant difference between the two injured groups occurred two to 24 weeks post injury (Table 2); However, this was not predictive of resolution of injury. 


\begin{tabular}{|c|c|c|c|c|c|c|}
\hline variable & $\begin{array}{l}2 \\
\text { weeks }\end{array}$ & $\begin{array}{l}4 \\
\text { weeks }\end{array}$ & $\begin{array}{l}8 \\
\text { weeks }\end{array}$ & $\begin{array}{l}12 \\
\text { weeks }\end{array}$ & $\begin{array}{l}16 \\
\text { weeks }\end{array}$ & $\begin{array}{l}24 \\
\text { weeks }\end{array}$ \\
\hline \multicolumn{7}{|l|}{ Tl group } \\
\hline$n=$ & 42 & 44 & 21 & 8 & 4 & 4 \\
\hline \multicolumn{7}{|l|}{ PI group } \\
\hline$n=$ & 6 & 7 & 8 & 9 & 7 & 10 \\
\hline 2PDT injured & 0.538 & 0.419 & 0.67 & 0.436 & 0.295 & 0.375 \\
\hline LT injured & 0.39 & 0.453 & 0.626 & 0.391 & 0.212 & 0.284 \\
\hline $\begin{array}{l}\text { altered } \\
\text { sensation }\end{array}$ & 0.287 & 0.441 & 0.281 & 0.712 & 0.378 & 0.568 \\
\hline FPC injured & 0.167 & 0.265 & 0.302 & 0.31 & 0.573 & 0.553 \\
\hline area & 0.208 & 0.659 & 0.64 & 0.662 & 0.62 & 0.934 \\
\hline
\end{tabular}

\section{Light touch pressure sensibility}

Due to the variability of the mean pressure threshold for the lateral dorsum of the tongue, this test lacked in sensitivity in identifying the injury in both groups. The permanent injury group displayed a significantly higher mean pressure sensibility at the injured site compared with the temporary injury group at two and four weeks (Table 2).

\section{Fungiform papillae count}

A significant reduction in papillae number on the injured side was seen at two to twelve weeks in this study (Table 1). The increase of fungiform papillae count as the injury resolved may explain the lack of difference in relation to the contralateral side after eight weeks of injury (Table 1). No difference in the number of fungiform papillae on the injured side of tongue was seen between the permanent injury and temporary injury groups at any stage (Table 2).

\section{S/B discrimination}

Normally a pin-prick is distinguishable on the lingual surface and all the contra lateral uninjured sides in this study responded to 5/5 sharp detection. Pin-prick sensation was variable in both permenant injury and temporal injury groups explaining its lack of sensitivity to nerve injury and no significant difference between the groups at any post-operative stage.

\section{Symptoms}

The incidence of altered sensation did not differ in the permenant injury group compared with the temporary injury group at any time post-injury (Table 2). However dysaesthesia was found only in the permenant injury group and troublesome altered sensation was more prevalent in this group due to increased reported rates of pain.

\section{Neuralgia}

Palpation induced neuralgia was significantly decreased in the permanent injury group compared with the temporary nerve injury group $(P=0.004$ Table 2$)$ at all stages.

\section{Predictive formulae}

Odds Ratios (ORs) were performed in order to predict the eventual outcome of the lingual nerve injury. Neuralgia, subjective function, two-point discrimination, light touch threshold and neuropathic area, at four weeks, were entered into multiple regression analysis. At eight weeks, subjective function, twopoint discrimination, light touch and area were entered into multiple regression analysis, and only subjective function was significant $(P=0.006$, OR 0.95). Thus, subjective function correctly predicted for $72 \%$ of permanent injuries at eight weeks postinjury. If the subjective function is over four (out of 10) this predicts for permanence of injury in this cohort. At 12 weeks no variables including, subjective function $(P=0.452)$ and neuropathic area $(P=0.079)$, were significant using multiple regression analysis. At 16, 24 and 52 weeks there were not sufficient patient data to carry out multiple regression analysis.

\section{DISCUSSION}

The conventional methods by which lingual nerve injuries are assessed are complicated, inconsistent, require specialist training or equipment, are time-consuming and are not suited to the clinic setting. Often the complexity of the assessment results in confusion and a delay in referring patients for specialist attention. As a result the necessary repair of these nerves is generally undertaken after a year, an unacceptable delay with respect to other peripheral nerve injuries. ${ }^{19}$ Simplification of the recommended assessment for lingual nerve injuries is imperative in order to ensure their recognition and expedite appropriate referral.

This study highlights that the application of many of these conventional mechanosensory tests in assessment of lingual nerve injury is limited. Only a few of these tests were able to reliably establish the presence of a nerve injury (neuropathic area, twopoint discrimination, symptoms and fungiform papillae count). This study also indicates that a simple semi-quantified subjective function test correlates well with two-point discrimination, light touch sensibility and neuropathic area subsequent to lingual nerve injuries. This supports previous reports that state if a patient reports a sensory problem there is a little chance that it can be verified using light touch, ${ }^{2}$ brush stroke methods or other the selected mechanosensory tests. ${ }^{13}$ This correlation of reported subjective function with 2PDT, neuropathic area and light touch, has been previously reported for inferior alveolar nerve injuries. ${ }^{21,22}$ Fridrich et al., ${ }^{21}$ also reported that as the injury resolved the patient's subjective function resolved quicker than the neurosensory tests. This may by due to the adjustment and habituation by the patient to their long standing injury, ${ }^{20}$ which may explain the reported normal function in the presence of mildly abnormal results as found in the present study for light touch sensibility and two-point discrimination test.

The pattern of sensory resolution seen in this investigation is similar to that found by Mason ${ }^{1}$ who reported that early presentation of partial injuries (only lateral border or tip of the tongue affected) were likely to resolve by three months and was highly significant for later recovery. This was substantiated in the present study, as there was a significant difference in the size of the neuropathic area between the temporarily and permanently injured groups at four, eight, 12 and 24 weeks, however the rate of reduction in the neuropathic area was not a predictive indicator for resolution of injury.

The incidence of altered sensation in this patient cohort was similar to other studies for both permanent and temporary injuries. ${ }^{6,23}$ The presence of altered sensation negatively correlated with subjective function at four, 12 and 24 weeks, possibly highlighting the increased rate of troublesome altered sensation in those with persistent injuries. Altered sensation provided no outcome predictive value, in agreement with a previous report. ${ }^{5}$ The presence of pain and spontaneous altered sensation was significantly higher in the permanent injury group, similar to the findings of Robinson et al. ${ }^{22}$ These authors reported that over $50 \%$ of 53 patients, presenting for lingual nerve repair with permanent injuries, suffered from spontaneous paraesthesia and 28\% also complained of frequent spontaneous pain.

The two-point discrimination thresholds were able to identify a nerve injury in this cohort of patients and thresholds were comparable with previous reports. 5,23,24 2PDT correlated with recovery of subjective function of lingual nerve injuries for the first 12 weeks post-injury. This has been previously reported with moving 2 PDT. ${ }^{5}$ Differentiation of permanent from temporary injuries occurred at 
four and eight weeks, with significantly greater 2PDT levels associated with permanent injuries. Despite this relative increase of 2PDT in the early post-injury, 2PDT was not an indicator for permanence of the injury, although the conventional clinical tests had the strongest correlation with subjective function.

The only other report of light touch pressure thresholds for injured and uninjured lingual nerves was carried out by Eliav and Gracely ${ }^{3}$ however, this was performed before and two or eight days after third molar surgery in only 12 patients. There is a distinct lack of baseline data for LT sensibility of the tongue despite the number of studies that report 'normal and abnormal' data. In the present report, due to the variability of LT pressure thresholds, the method only discriminated between injured and contralateral sides at four weeks post-injury. This highlights the lack of "within patient' sensitivity of this test for lingual nerve injury and inability to differentiate permanent from temporary injury.

The results of the present study showed sharp, blunt discrimination to be the least discriminatory in comparison with the other assessments in contrast to two previous reports. ${ }^{22,23}$ Estimation of fungiform papillae number was sensitive to detecting nerve injury similar to previous reports. ${ }^{21}$ However, it was unable to distinguish subjects with temporary or permanent lingual nerve injuries early post-injury. The mechanism for a reduction in papillae number is their dependence on neurotrophins, the reduction of which subsequent to injury to the chorda tympani causes lack of papillae trophism. ${ }^{25}$

Elicitation of neuralgic type pain by palpation of the lingual nerve in the retromolar region has been reported to be associated with neuroma formation ${ }^{26}$ and described as an indication for surgical exploration of the lingual nerve. However in this study, palpation induced neuralgia was significantly higher in the temporary injuries compared with the permanent injuries.

This investigation is the first to attempt to quantify patients' reported lack of function, associated with lingual nerve injury using a simple standardised patient centred clinical test, so there are no comparative data from previous reports. This subjective function test assumes that there is no change in thresholds contralateral to the injury; this may, however, not be the case as CNS neuroplasticity may result in elevated thresholds on the contralateral side. A possible limitation of this subjective function assessment method is in those patients suffering from allodynia; as a result the subjective score may be increased rather than decreased, not necessarily reflecting the reduced functional capacity. However, despite this limitation, subjective function proved to be correctly predictive of temporary (87-88\%) and permanent injuries (66-72\%) at four and eight weeks in this cohort of patients. The use of a stiff von Frey 12 fibre for this test provides a standardised stimulus and results in supra threshold stimulation and substantial indentation of the mucosa on application, similar to using a periodontal probe or closed calliper tips by multiple investigators deeming it a suitable test for general practice. ${ }^{27}$

\section{CONCLUSION}

This study attempted to clarify the relationship between patient reports and clinical neurosensory testing of the lingual nerve. Although palpation induced neuralgia, subjective function, 2PDT and neuropathic area all showed a significant difference early post-injury between temporary and permanent injuries. Reduced subjective function was the only test to differentiate between the groups and predict for outcome at four and eight weeks post injury. A more sophisticated quality of life assessment, using previously validated questionnaires regarding the various aspects of quality of life (oral and social function, anxiety and depression and health belief), may prove to be a more accurate disability assessment for these patients and would be advantageous as an outcome measure for medical or surgical intervention and for comparison with better known nerve injury or pain conditions.

The subjective method of assessment (subjective score) alone may be sufficient in assessing patients with lingual nerve injury, at least in the early post-operative phase. Suitable for general practice or hospital setting, it has the added advantage that it requires minimal time, expertise or specialist equipment. This should enable the non-specialist and specialist alike to identify those patients at risk of permanent injury and to arrange suitable referral of the patient for specialist assessment and possible reparative surgery.

In summary the clinical tests which were sensitive to recovery of lingual nerve injury (most sensitive to least sensitive) were:

- subjective function $>$ neuropathic area $>2 \mathrm{PDT}>\mathrm{LT}>\mathrm{FPC}>\mathrm{S} / \mathrm{B}$ $=$ altered sensation $=$ neuralgia $=$ mucosal scarring.

- prediction of permanence of lingual nerve injury may be based on two simple tests:

- lowered subjective function $<4(0-10)$, thus avoiding complex neuroassessment

- Identification of an injury was best achieved using the:

- subjective function test >neuropathic area>2PDT test.

We would like to thank the patients that agreed to take part in this study.

1. Mason D A. Lingual nerve damage following lower third molar surgery. Int J Oral Maxillofac Surg 1988; 17: 290-294

2. de Beukelaer J G P. Smeele LE, van Ginkel F C. Is short-term testing after removal of mandibular third molars efficacious? Oral Surg Oral Med Oral Pathol Oral Radiol Endod 1998; 85:366-370.

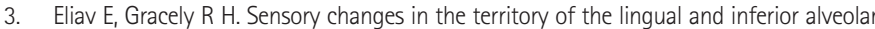
nerves following lower third molar extraction. Pain 1998: 77: 191-199.

4. Shafer D M, Frank M E, Gent J F, Fischer M E. Gustatory function after third molar extraction. OralSurg Oral Med Oral Pathol Oral Radiol Endod 1999; 87: 419-428.

5. Blackburn C W. A method of assessment in cases of lingual nerve injury. Br J Oral and Maxillofac Surg 1990; 28: 238-245.

6. Schultze-Mosgau S, Reich R H. Assessment of inferior alveolar and lingual nerve disturbances after dentoalveolar surgery, and recovery of sensitivity. Int J Oral Maxillofac Surg 1993; 22: 214-217.

7. Teerijoki-Oksa $T$, Jaaskelainen $S$, Forsell $K$, Forsell $H$. An evaluation of clinical and electrophysiologic tests in nerve injury diagnosis after mandibular sagittal split osteotomy. Int J Oral and MaxilofacialSurgery 2003; 32: 15-23.

8. Feldman J A, Essick G K, Zuniga J R, Phillips C. Interexaminer reliability of three subjective neurosensory tests. Int JAdult Orthod Orthognath Surg 1997; 12: 273-285.

9. Ylikontiola L, Kinnunen J, Oikarinen K. Comparison of different tests assessing neurosensory disturbances after bilateral sagittal split osteotomy. Int J Oral Maxillofac Surg 1998; $27: 417-421$

10. Ylikontiola L, Kinnunen J, Laukkanen $P$, Oikarinen K. Prediction of recovery from neurosensory deficit after bilateral sagittal split osteotomy. Oral Surg Oral Med Oral Path Radiol Endod 2000; 90: 275-281.

11. Zuniga $J R$, Meyer $R A$, Gregg $J M$, et al. The accuracy of clinical neurosensory testing for nerve injury. J Oral Maxillofac Surg 1998; 56: 2-8.

12. Campbell R L, Shamaskin R G, Harkins SW. Assessment of recovery from injury to inferior alveolar and mental nerves. Oral Surg Oral Med Oral Path 1987; 64: 519-526.

13. Cunningham $L \mathrm{~L}$, Tiner $B$, Clark $G M$ et al. A comparison of questionnaire versus monofilament assessment of neurosensory deficit. J Oral Maxillofac Surg 1996; 54: 454459

14. Robinson P P, Smith K G. Lingual nerve damage during lower third molar removal: a comparison of two surgical methods. BrDentJ 1996; 180: 456-461.

15. Scrivani S J, Moses M, Donoff R B, Kaban L B. Taste perception after lingual nerve repair. J Oral Maxillofac Surg 2000; 58: 3-5

16. Gregg J M. Posttraumatic pain. Experimental trigeminal neuropathy. J Oral Surg 1971; 29: 260-267.

17. Mackinnon S E, Dellon A L. Surgery of the peripheral nerve. Pp 35-66. New York: Thieme Medical Publishers, 1988

18. Bell-Krotoski J, Tomancik E. The repeatability of testing with Semmes-Weinstein monofilaments. J Hand Surg 1987; 12A: 155-161.

19. Zuniga J R, Essick G K. A contemporary approach to the clinical evaluation of trigemina nerve injuries. Oral Maxillofac Surg Clin Nth America 1992; 4: 353-357.

20. Birch R, Bonney G, Wynn Parry C B. Surgical disorders of the peripheral nerves. Edinburgh: Churchill-Livingstone, 1998

21. Fridrich K L, Holton T J, Pansegrau K J, Buckley M J. Neurosensory recovery following the mandibular bilateral sagittal split osteotomy. J Oral Maxfac Surg 1995; 53: 1300-1306.

22. Chen N, Yang X, Zuniga J R. Quantitative studies of taste and fungiform papillae on the anterior human tongue. Zhonghua Kou Qiang Yi Xue Za Zhi 1998; 33: 140-142. (author's translation)

23. Robinson $P P_{1}$, Loescher $A R$, Smith $K G$. A prospective quantitative study on the clinical outcome of lingual nerve repair. Br J Oral Maxillofac Surg 2000; 38: 255-263.

24. Joshi A, Rood J P. External neurolysis of the lingual nerve. Int J Oral Maxillofac Surg 2002; 31: 40-43.

25. Cowan PW. Atrophy of fungiform papillae following lingual nerve damage - a suggested mechanism. BrDent J 1990; 168: 95.

26. Zuniga J R. Multi modal scaling of sensory recovery. J Oral Maxillofac Surg 1990; 48: 85-89.

27. Dellon A L, Mackinnon S E, Crosby P M. Reliability of two-point discrimination measurements. JHand Surg 1987: 12A: 693-636. 